\title{
Measurement of cosmic-ray charge with the DAMPE Silicon-Tungsten Tracker
}

\section{S. Vitillo*1 and V. Gallo1, on behalf of the DAMPE collaboration}

${ }^{1}$ Département de Physique Nucléaire et Corpusculaire, Université de Genève, 1211 Geneva, Switzerland

E-mail: stefania.vitillo@cern.ch

The DArk Matter Particle Explorer (DAMPE) satellite is a powerful space detector launched on December $17^{\text {th }} 2015$. The main objectives of the mission are in the research for Dark Matter signatures thanks to the detection of electrons and photons in an energy range going from few $\mathrm{GeV}$ up to $10 \mathrm{TeV}$. Moreover, insights on the origin and propagation mechanisms of cosmic rays are also expected thanks to nuclei flux measurements up to $100 \mathrm{TeV}$. In this context the charge (Z) measurement is crucial to distinguish the different chemical components of cosmic rays and to check the models of propagation in the galaxy. The DAMPE detector is composed of the following sub-detectors: a Plastic Strip scintillator Dectector (PSD), a Silicon-Tungsten tracKer (STK), a Bismuth Germanium Oxide (BGO) imaging calorimeter, and a NeUtron Detector (NUD). The Z-measurement is performed by the PSD and the STK. In this document after a detailed description of the STK, the first results on the absolute ion charge identification using more than one year of STK on-orbit data are discussed, together with the applied calibration procedure.

35th International Cosmic Ray Conference - ICRC2017

10-20 July, 2017

Bexco, Busan, Korea

\footnotetext{
* Speaker.

${ }^{\dagger}$ The DAMPE mission is funded by the strategic priority science and technology projects in space science of Chinese Academy of Sciences. In Europe is supported by the Swiss National Science Foundation (SNSF) and the National Institute for Nuclear Physics (INFN), Italy.
} 


\section{The DArk Matter Particle Explorer (DAMPE)}

DAMPE [1] was successfully launched into a sun-synchronous orbit at the altitude of $500 \mathrm{~km}$ on December $17^{\text {th }} 2015$. The objectives of the DAMPE experiment are the searches for Dark Matter decay or annihilation signatures, gamma-ray astronomy and the measurement of cosmic-ray fluxes. DAMPE provides multiple measurements in order to identify the crossing particle thanks to different subdetectors: a double-layer Plastic Scintillator strip Detector (PSD) [2] used for the measurement of the absolute charge $(\mathrm{Z})$ and as anti-coincidence for photons; a Silicon-Tungsten tracKer-converter (STK) that reconstructs the trajectory of the particle [3], measures its IZI and converts incoming photons into electron-positron pairs to estimate the photon direction; a Bismuth Germanium Oxide imaging calorimeter (BGO) of about 32 radiation lengths for precise energy measurements and for electron/photon identification [4]; a NeUtron Detector (NUD) to improve the electron/proton separation power. The detector layout is shown in Fig. 1.

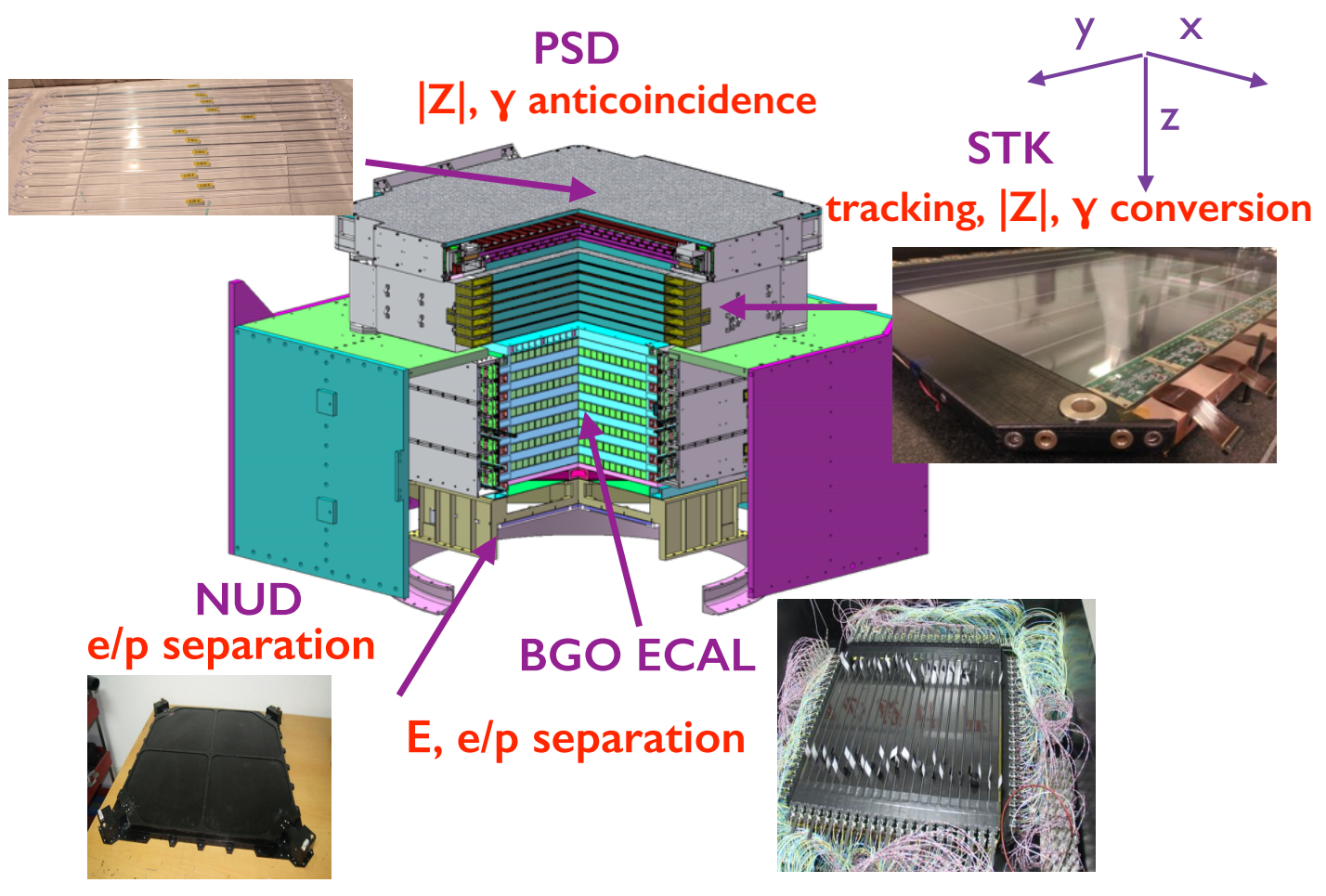

Figure 1: Layout of the DAMPE experiment.

\section{The Silicon-Tungsten tracKer-converter (STK)}

The STK consists of a total of 7 planes with 6 silicon layers reading the $\mathrm{x}$ coordinate, 6 silicon layers reading the y coordinate of the particle, forming so 6 tracking double layers. Each tracking layer is equipped with single sided AC-coupled Silicon micro-Strip Detectors (SSD), placed to measure the two orthogonal views perpendicular to the pointing direction of the apparatus ( $\mathrm{z}$ axis in Fig. 1). To allow photon conversion, three layers of tungsten plates with thickness of $1 \mathrm{~mm}$ are 
inserted into the support trays of the second, third and fourth tracker plane. A schematic view is shown in Fig. 2. Each silicon layer is made of 16 ladders, each formed by 4 SSD for a total of 192 ladders. The sensors are $320 \mu \mathrm{m}$ thick, $9.5 \times 9.5 \mathrm{~cm}^{2}$ in surface, and segmented into 768 strips with $121 \mu \mathrm{m}$ pitch. In order to limit the number of read-out channels and keep a good performance in terms of space resolution [5] the read-out is done only every other strip for a total of 384 channels per ladder that are read by 6 VA140 ASIC chips (Fig. 3).

The STK has been successfully built in spring 2015. A picture of the tracker before the assembly of the last tray, the front-end electronics and the cooling and supporting structures is shown in Fig. 4.

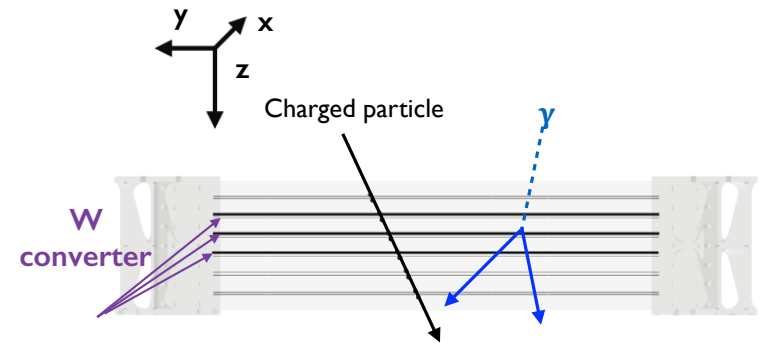

Figure 2: Schematic view of the behavior of different particles traversing the STK.

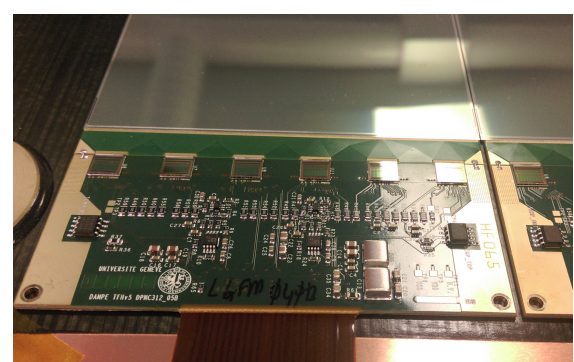

Figure 3: Picture of the edge of one ladder readout by 6 VA.

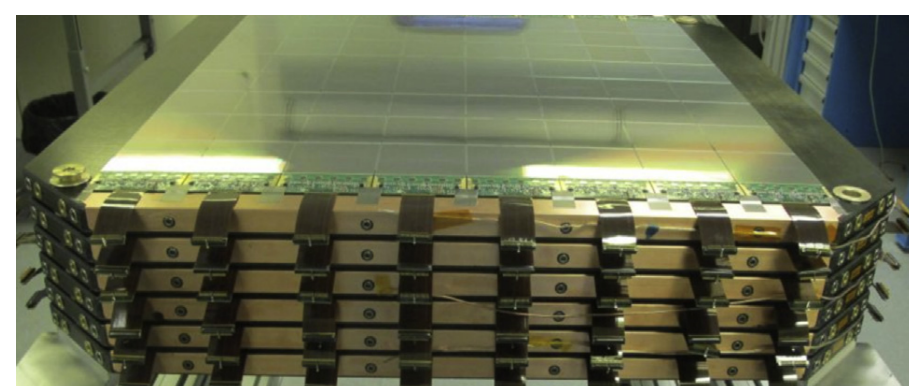

Figure 4: Picture of the STK before the assembly of the last tray. The 16 ladders forming one sensitive layer of the STK are clearly visible.

The deposited ionization energy in a silicon sensor is proportional to the square of the particle charge ( $d E / d x \propto Z^{2}$ ), therefore it allows to distinguish different nuclear species. In the following, the charge calibration procedure for proton and helium nuclei is described.

\subsection{Charge Measurement}

The ionization energy loss in a silicon sensor is collected by a cluster of adjacent read-out strips. The cluster amplitude is defined as the sum of each single-cluster strip signal. A threshold applied to the strip signal to noise ratio defines a valid signal. The cluster amplitude depends on the impact position, on the charge of the particle and on its impact angle. With the help of the tracking algorithm (described in [6]) only clusters belonging to a track are considered in the following. The impact position of the particle on the silicon is estimated directly from the cluster signal read-out 
through the variable $\eta$ defined as:

$$
\eta=\frac{S_{1}}{S_{1}+S_{2}}
$$

where $S_{1}$ and $S_{2}$ are the amplitudes of the two channels with highest signal, identified by their readout order. Fig. 5 shows the relation between $\eta$ and the impact position. The charge relative to $\mathrm{p}$ and He was selected from the combined information of the two layers of PSD, after correcting for the path length of the particle, with the variable:

$$
\mathrm{PSD}_{\text {global }}=\sqrt{\frac{\mathrm{PSD}_{\mathrm{x}}+\mathrm{PSD}_{\mathrm{y}}}{2 \cdot \mathrm{E}_{\mathrm{MIP}}}}
$$

where $\mathrm{E}_{\mathrm{MIP}}$ is the value of the energy that a minimum ionizing particle loses in $10 \mathrm{~mm}$ of PSD (for accurate parametrization and details see [7]). The distribution of $\mathrm{PSD}_{\text {global }}$ is shown in Fig. 6. Proton and helium candidates are selected requiring $\operatorname{PSD}_{\text {global }}(\mathrm{p}) \in[0.94,1.24]$ and $\operatorname{PSD}_{\text {global }}(\mathrm{He}) \in$ $[2.04,2.26]$. The impact angle on the $\mathrm{xz}\left(\theta_{\mathrm{x}}\right)$ and $\mathrm{yz}\left(\theta_{\mathrm{y}}\right)$ views are obtained from the reconstructed track in STK.

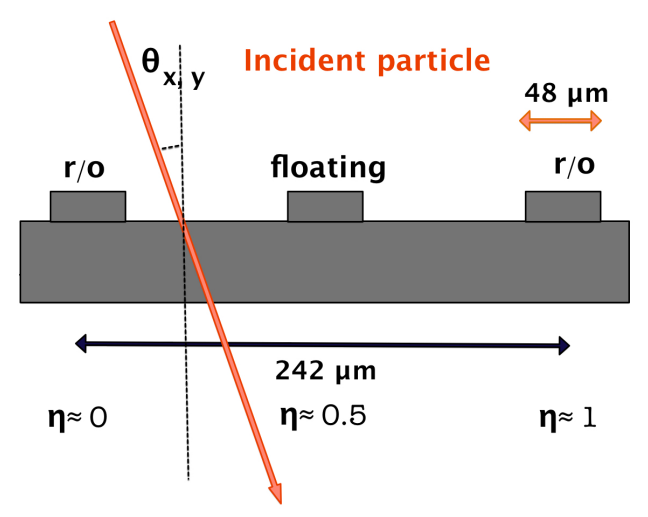

Figure 5: Schematic view of the relation between $\eta$ and the particle impact point; r/o (read-out) and floating correspond to three consecutive strips of one ladder.



Figure 6: $\mathrm{PSD}_{\text {global }}$ distribution for proton and helium; the red delimited distribution represents the selected proton and helium nuclei.

\subsection{Charge Selection}

In order to select particles crossing the STK detector, a sample of events containing tracks with 5 or 6 reconstructed points is selected. The cluster charge distribution after path length correction and the VA gain correction (described in [8]) of ladders belonging to the first two STK layers is shown in Fig. 7 for $Z=1$ and for $Z=2$, and for two different impact angles.

In the 3-dimensional plots of Fig. 7, the black points correspond to the value where the Landau convoluted with a Gaussian noise function, that was used to fit the energy loss distribution for different values of $\eta$ (examples for proton in Fig. 8, for helium in Fig. 9), is maximum. We can notice different signal values depending on $\eta$ for different particles. A smooth spline function connects the points to show the behavior. This dependence is a consequence of a loss of collection efficiency when a particle crosses the sensor in between two read-out strips, while the maximum charge collection efficiency is obtained when the particle impacts vertically on a read-out strip. At steeper inclinations, the signal tends to be distributed in a larger number of strips which partially 

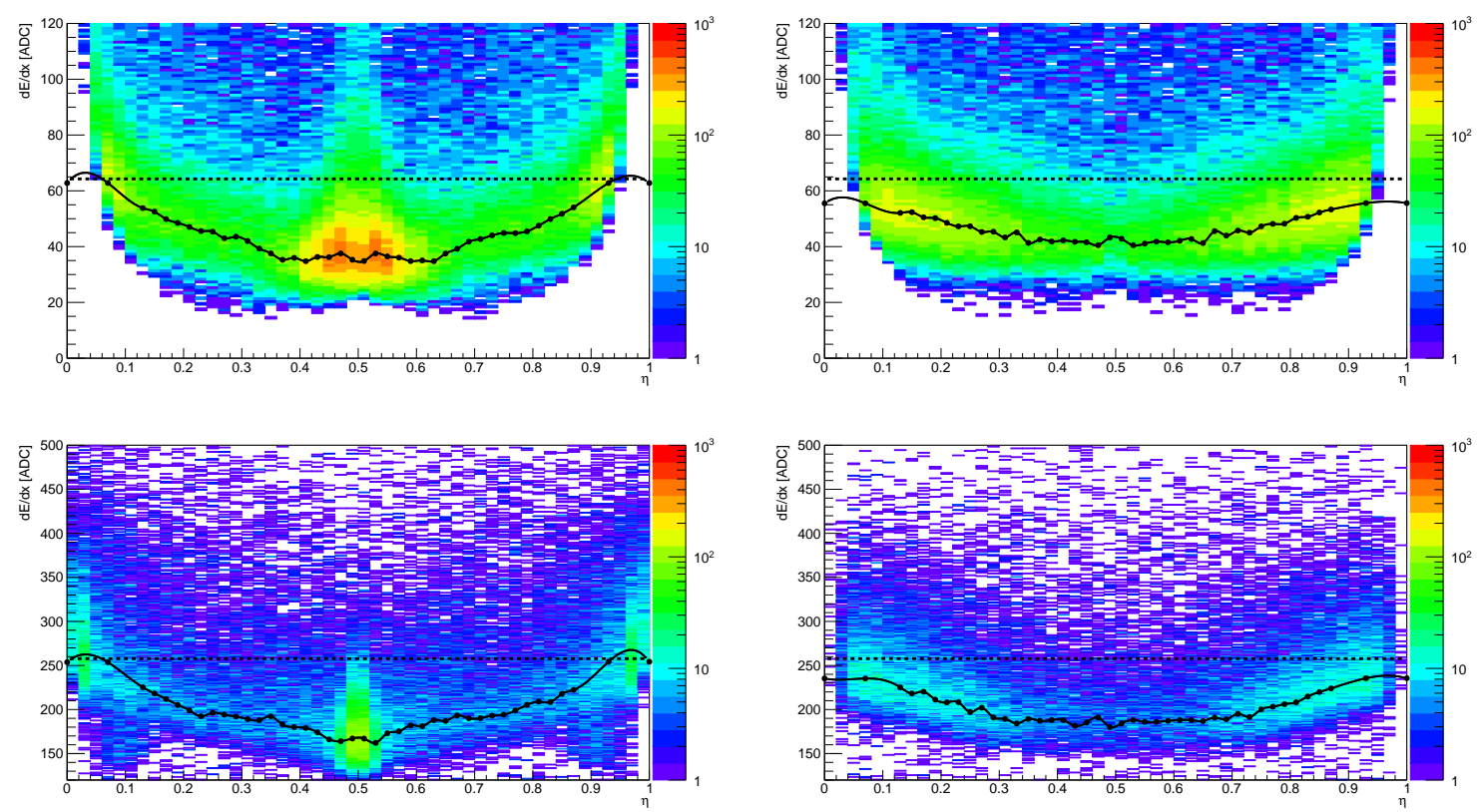

Figure 7: Energy loss as function of $\eta$ for a selected sample of protons (top) and helium nuclei (bottom) for $5^{\circ}<\left|\theta_{x, y}\right|<10^{\circ}$ (left) and $25^{\circ}<\left|\theta_{x, y}\right|<30^{\circ}$ (right). The dashed line corresponds to the value of the energy loss at which we want to correct.

compensates for the loss of collection and attenuates the effect. The dashed line corresponds to the value of the energy loss at which we want to correct (the energy loss for particles that impinge on read-out strips vertically).
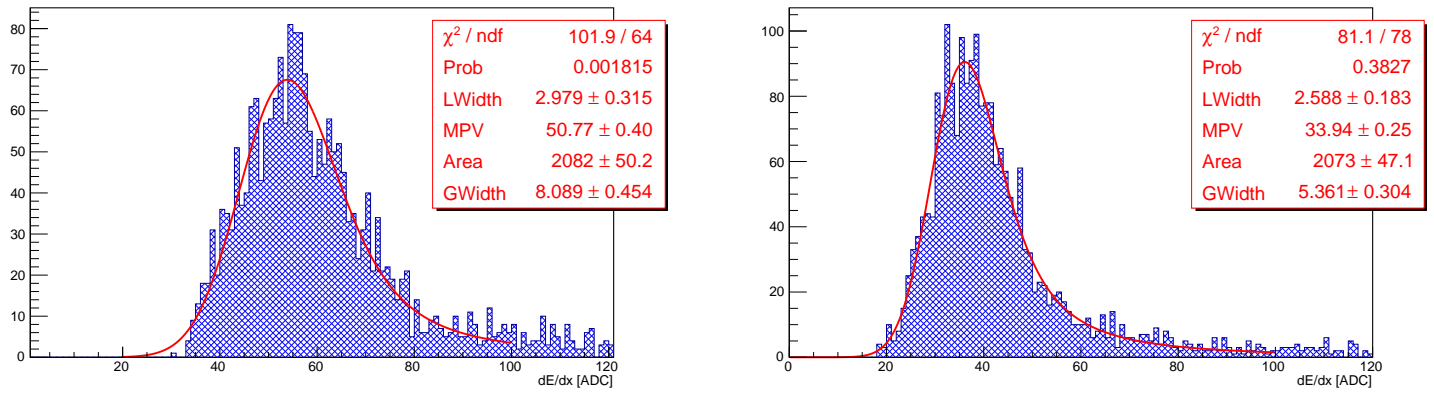

Figure 8: Energy loss for proton for $\eta \in[0.12,0.14]$ (left) and $\eta \in[0.38,0.40]$ (right) both for $5^{\circ}<\left|\theta_{x, y}\right|<$ $10^{\circ}$. A fit with a Landau convoluted with a Gaussian noise function is also shown in both cases. Note the different collection efficiency as function of $\eta$.

\subsection{Charge Loss Correction}

With the energy distribution fit results (shown for example in Fig. 8) correction factors for proton and helium in the first point of STK were computed for various inclinations within the range $0^{\circ}<\left|\theta_{x, y}\right|<35^{\circ}$ with $5^{\circ}$ step as:

$$
\operatorname{corr}_{\text {param }}=\operatorname{Max}_{\mathrm{dE} / \mathrm{dx}}\left(\mathrm{Z}_{\mathrm{i}}\right) / \operatorname{fun}\left(\eta_{\mathrm{Z}_{\mathrm{i}}}, \theta_{\mathrm{x}, \mathrm{y}}\right)
$$



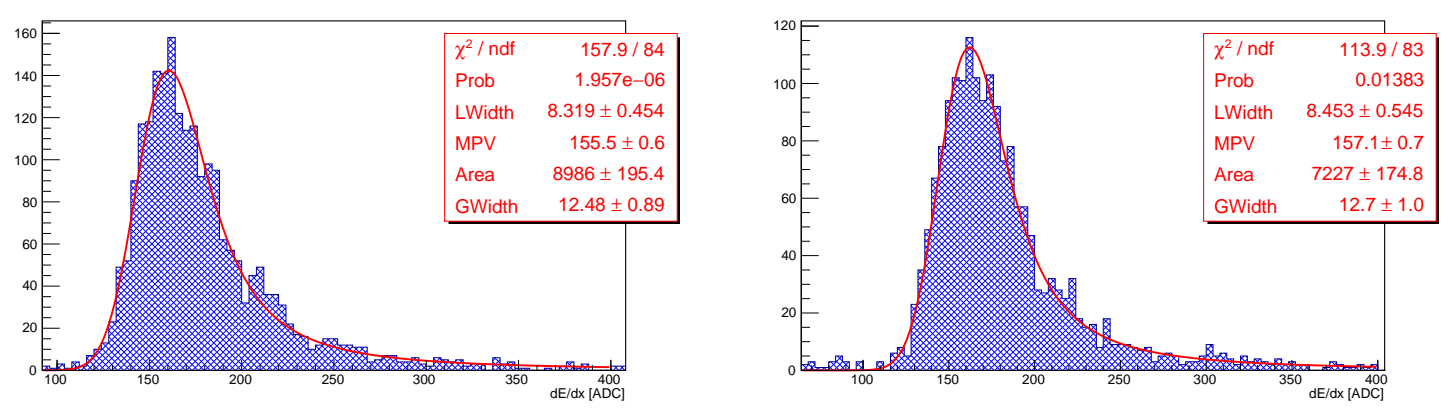

Figure 9: Energy loss for helium for $\eta \in[0.52,0.54]$ and $\left|\theta_{x, y}\right|<5^{\circ}$ (left) and $5^{\circ}<\left|\theta_{x, y}\right|<10^{\circ}$ (right). A fit with a Landau convoluted with a Gaussian noise function is also shown.

where $\operatorname{Max}_{\mathrm{dE} / \mathrm{dx}}\left(Z_{\mathrm{i}}\right)$ is the value where the Landau convoluted with a Gaussian noise function for $\eta \in[0,0.12]$ and for $\left|\theta_{x, y}\right|<5^{\circ}$ is maximum for proton or helium $\left(Z_{\mathrm{i}}\right)$ particles and $\operatorname{fun}\left(\eta_{\mathrm{Z}_{\mathrm{i}}}, \theta_{\mathrm{x}, \mathrm{y}}\right)$ is the spline function evaluated at $\eta$ for the inclination $\theta_{x, y}$. We applied these correction parameters to the energy distribution of all the 192 STK ladders. We also applied a cut on the cluster charge in order to reduce particles converting with STK and the presence of misidentified protons in PSD.

\section{Charge Identification Performances}

Fig. 10 shows the energy loss distribution for protons and helium nuclei in all the ladders before and after the energy loss correction. The improvement in the cluster charge shape is evident.
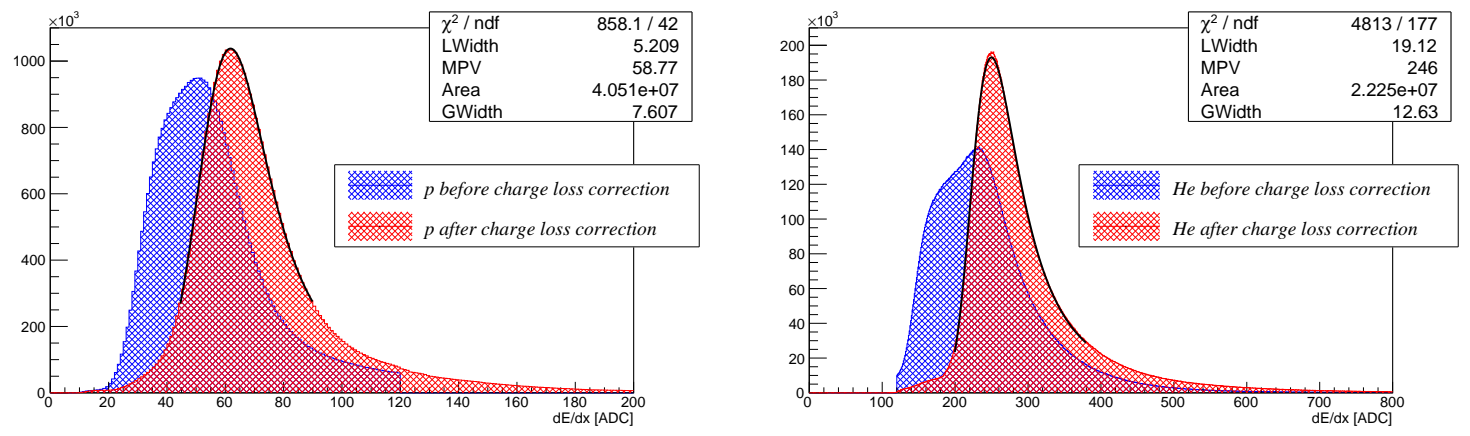

Figure 10: Energy loss distribution for protons (left) and helium nuclei (right) in the STK ladders before (blue) and after (red) the charge loss correction. A fit with a Landau convoluted with a Gaussian noise function for the corrected distribution is also shown for completeness.

To have an averaged charge value that combines all the measurements of the clusters belonging to the track, associated to the charged particle that we have selected, we used $S_{T}$ defined as:

$$
S_{T}=\frac{\sum_{i=1}^{\# c l u} S_{i}-S_{\text {Max }}}{\# c l u-1}
$$

where $S_{M a x}$ is the maximum signal among the clusters associated to the track. The square root of the truncated mean calculation is used for its direct proportionality to the charge of the particle 
$\left(\sqrt{S_{T}} \propto Z\right)$. We divided $\sqrt{S_{T}}$ by the square root of the signal for protons and its distribution before and after the charge loss correction is shown in Fig. 11.

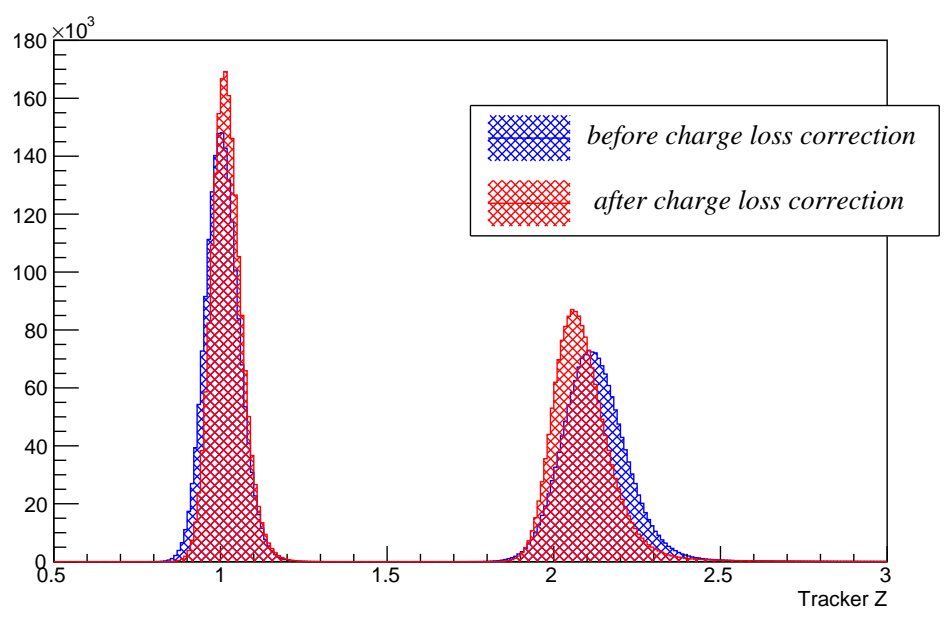

Figure 11: Tracker charge measurement before (blue) and after (red) applying the charge loss correction.

\section{Conclusions}

In cosmic-ray flux measurements redundancy of the absolute charge identification is very important and an additional measurement of the charge has to be performed with highest precision as possible. By fitting the charge distribution in Fig. 11 with a Gaussian function for each charge after the charge loss correction, we obtained the preliminary measurement of the charge resolution for protons and helium nuclei in the STK in charge units (c.u.): $\sigma_{\mathrm{p}}=0.04$ c.u. and $\sigma_{\mathrm{He}}=0.07$ c.u. The statistical uncertainties of the fits are very small and are negligible. Charge resolutions after charge loss correction have improved by $\approx 13 \%$ for protons and $\approx 21 \%$ for helium nuclei and can improve the measurement of the proton flux [9] and helium flux with DAMPE [10].

\section{References}

[1] J. Chang et al. (DAMPE Collaboration), The Dark Matter Particle Explorer mission, Astropart. Phys. submitted (2017) [arXiv:1706.08453].

[2] Y. Yu et al. The Plastic Scintillator Detector at DAMPE, [arXiv:1703.00098], 2017

[3] P. Azzarello et al. The DAMPE silicon-tungsten tracker Nucl. Instrum. Meth. A 831 379-384, 2016

[4] Z. Zhang et al. The calibration and electron energy reconstruction of the BGO ECAL of the DAMPE detector, Nucl. Instrum. Meth. A 836 (2016) 98 [arXiv:1602.07015].

[5] A.Tykhonov et.al. (DAMPE collaboration) Internal alignment and position resolution of the silicon tracker of DAMPE determined with the orbit data to be submitted to Nucl. Instrum. Meth. A

[6] A.Tykhonov et.al. Reconstruction software of the silicon tracker of DAMPE mission in proceeding of CHEP 2016, accepted to Journal of Physics Conference Series (JPCS) 
[7] Y. Zhang et al. PSD performance and charge reconstruction with DAMPE in proceedings of The 35th International Cosmic Ray Conference, POS ( ICRC2017) 1682017

[8] V. Gallo et al. The DAMPE silicon tungsten tracker in proceedings of The 25rd International Workshop on Vertex Detectors, POS (Vertex2014) 0302016

[9] C. Yue et al. Studies on cosmic-ray proton flux with DAMPE in proceedings of The 35th International Cosmic Ray Conference, PoS ( ICRC2017) 10762017

[10] V. Gallo et al. Studies on Helium flux with DAMPE in proceedings of The 35th International Cosmic Ray Conference, POS ( ICRC2017) 1692017 